\title{
Withdrawal seizure associated with high dosage of aripiprazole and fluoxetine: a case report
}

\author{
Jian-An Su ${ }^{1,2,3^{*}}$, Shih-Yong Chou ${ }^{1,2,3}$, Ching-Shu Tsai ${ }^{1,2,3}$, Tai-Hsin Hung ${ }^{1,2,3}$ \\ ${ }^{1}$ Department of Psychiatry, Chang Gung Memorial Hospital, Chiayi, Chinese Taipei; \\ ${ }^{2}$ Graduate Institute of Clinical Medical Sciences at Chang Gung University, Taoyuan, Chinese Taipei; \\ ${ }^{3}$ Department of Nursing, Chang Gung Institute of Technology, Taoyuan, Chinese Taipei. \\ E-mail: sujian@adm.cgmh.org.tw
}

Received 13 May 2011; revised 22 June 2011; accepted 30 June 2011.

\begin{abstract}
Aripiprazole, a third-generation antipsychotic, has been considered to have a high safety profile and rare withdrawal symptoms. We reported the case of a schizophrenic patient with a significant obsession, who was treated with a high dosage of aripiprazole and fluoxetine. Generalized tonic-clonic seizure occurred two days after abruptly stopping these two medications. Gradually tapering aripiprazole is suggested in clinical practice, especially when using a high dosage.
\end{abstract}

Keywords: Aripiprazole; High Dosage; Withdrawal Seizure

\section{INTRODUCTION}

Aripirazole is a so-called third-generation antipsychotic with a unique pharmacological mechanism [1,2]. It has indications for the treatment of schizophrenic and bipolar patients $[3,4]$ and is also effective in augmenting antidepressants in neurotic patients including major depressive disorder and obsessive compulsive disorder [5-7]. Its safety and efficacy are already well-established [3,8], and few withdrawal symptoms have been reported in clinical trials. Herein, we present the case of a patient with schizophrenia, paranoid type, with significant obsession and delusion. High-dosage aripiprazole $30 \mathrm{mg}$ and fluoxetine $60 \mathrm{mg}$ were prescribed concurrently for three weeks for symptom relief. Generalized tonic-clonic seizure occurred two days after abruptly stopping the two medications.

\section{CASE REPORT}

This 40-year-old male patient had been diagnosed with paranoid schizophrenia for about 10 years. He took haloperidol $4 \mathrm{mg}$ and zotepine $50 \mathrm{mg}$ per day, but the response was incomplete remission. The patient still had residual psychotic symptoms including delusion of persecutory and reference under these regimens. In addition, the gradual exacerbation of significant obsession, sexual image intuition, was impacting his quality of life. Aripiprazole was prescribed and combined with fluoxetine to help control of his delusions and obsession. The score of clinical global impression-severity (CGI-S) was 5 at that time. We arranged for aripiprazole $10 \mathrm{mg}$ to be taken in the first week, which was then titrated to aripiprazole 20 $\mathrm{mg}$ and we also added on fluoxetine $40 \mathrm{mg}$ in the second and third weeks. Because the patient's psychotic symptoms and obsession still persisted, we escalated the dosage to aripiprazole to $30 \mathrm{mg}$ and fluoxetine to $60 \mathrm{mg}$. The symptoms showed little improvement after one week on this high-dose regimen and his delusions even worsened. In addition, the patient also complained of akathisia. Therefore, we abruptly stopped the above two medications and set up olanzapine $10 \mathrm{mg}$ specifically for his exacerbated delusions. However, generalized tonic-clonic seizure was noted the second day after stopping aripiprazole and fluoxetine. Head injury with subarachnoid hemorrhage occurred because the patient lost consciousness and fell down during a seizure. He was admitted to the Neurosurgery Ward for observation and a survey of the seizure etiologies, but no remarkable abnormality was detected, including the electroencephalography (EEG) results. The family denied a past history or family history of seizure attack. After discharge, the medication was maintained at olanzapine $10 \mathrm{mg}$, haloperidol $5 \mathrm{mg}$ and escitalopram $30 \mathrm{mg}$, concurrently, for his obsession and psychotic symptoms. Under this regimen, his condition gradually improved. An EEG was arranged again four months after discharge, and still there was no abnormality noted.

\section{DISCUSSION}

This case involved a seizure that occurred after abruptly discontinuing a high dosage of aripiprazole and fluoxe- 
tine. This is the first reported case of a withdrawal seizure related to the abrupt discontinuation of both aripiprazole and fluoxetine in combination. There are some possible explanations for the emergence of a seizure attack in this patient, including aripiprazole withdrawal, fluoxetine withdrawal, or induction by olanzapine. Aripiprazole is supposed to be at low-risk for seizure attack and the reported risk is $0.1 \%$ [9]. Only two cases of seizure during treatment with aripiprazole have been reported in the related literature and the daily dosage was $10 \mathrm{mg}$ and $15 \mathrm{mg}$ respectively [10,11]. Aripiprazole has the unique effect of dopamine stabilization, and also has a partial 5-HT1 agonist effect and antagonist activity at 5-HT2A receptors [1]. The mechanism of partial agonist effect in 5-HT1 provides efficacy, not only in treating psychotic symptoms, but also in augmenting antidepressants in the treatment of depression and obsession-compulsion [5-7]. The combination of aripiprazole and fluoxetine seemed appropriate for this case. However, fluoxetine is a cytochrome P450 (CYP) 2D6 inhibitor and aripiprazole is metabolized mainly by CYP2D6 [12]. This patient had taken the maximum recommended daily dosage of aripiprazole; however, the serum concentration may have been higher than we expected because of the inhibition of CYP2D6 by fluoxetine. Withdrawal seizure may occur when abruptly stopping such a high-level concentration of aripiprazole.

Fluoxetine has been marketed much longer than aripiprazole. The possibility of fluoxetine-induced seizure attack was around $0.1 \%$ in randomized controlled trials, and is classified as low-risk among antidepressants [13]. It has been a report of a seizure protective effect in an animal model [14]. Fluoxetine has a long half-life for 24 to 48 hours [12]. Withdrawal symptoms are fewer, compared to other antidepressants [15], and no fluoxetine withdrawal seizure has been reported so far. Thus, the seizure attack in this case more likely resulted from aripiprazole withdrawal than fluoxetine withdrawal, especially with the high concentration of aripiprazole. In most drug trials with aripiprazole, the dosage usually fell between 15 and $30 \mathrm{mg}$, and the patients seemed to tolerate this dosage well [16]. No severe withdrawal symptoms have been reported. Some adult patients have taken high-dosage aripiprazole, 45 to $75 \mathrm{mg}$ per day, and all of them seemed to tolerate it well [17-19]. However, an adolescent who took $60 \mathrm{mg}$ aripiprazole and $60 \mathrm{mg}$ fluoxetine concurrently per day for at least three weeks revealed a blunted affect, sluggishness, and cognitive slowing [20]. These reports described the patients' condition under a high dosage of aripiprazole, so we had no idea if any withdrawal symptoms would occur when stopping such a high dosage abruptly.

This patient's seizure attack occurred on the second day after switching to olanzapine. Olanzapine probably lowered the seizure threshold [21]; the pre-marketing seizure incidence for olanzapine was about $0.9 \%$ [22]. However, this patient continued olanzapine for three months after the seizure, and no other attacks occurred. An olanzapine-induced seizure attack was therefore less likely in this case.

\section{CONCLUSIONS}

Aripiprazole is very frequently combined with antidepressants in clinical practice such as augmentation for refractory major depressive disorder or obsessive compulsive disorder. The potential 2D6 suppression by the antidepressants might increase the serum level of aripiprazole. Withdrawal seizure might occur when abruptly discontinuing treatment, if the patient has taken high doses of aripiprazole with an antidepressant. Thus, gradual tapering is quite important to avoid possible withdrawal symptoms including seizure attack.

\section{REFERENCES}

[1] Burris K.D., Molski, T.F., Xu, C., Ryan, E, Tottori, K., Kikuchi, T., Yocca, F.D. and Molinoff, P.B. (2002) Aripiprazole, a novel antipsychotic, is a high-affinity partial agonist at human dopamine $\mathrm{D}_{2}$ receptors. The Journal of Pharmacology and Experimental Therapeutics, 302, 381-389. doi:10.1124/jpet.102.033175

[2] Naber, D. and Lambert, M. (2004) Aripiprazole: A new atypical antipsychotic with a different pharmacological mechanism. Progress in Neuropsychopharmacology \& Biological Psychiatry, 28, 1213-1219.

doi:10.1016/j.pnpbp.2004.06.020

[3] Potkin, S.G., Saha, A.R., Kujawa, M.J., Carson, W.H., Ali, M., Stock, E., Stringfellow, J., Ingenito, G. and Marder, S.R. (2003) Aripiprazole, an antipsychotic with a novel mechanism of action, and risperidone vs. placebo in patients with schizophrenia and schizoaffective disorder. Archives of General Psychiatry, 60, 681-690. doi:10.1001/archpsyc.60.7.681

[4] Keck, P.E., Orsulak, P.J., Cutler, A.J., Sanchez, R., Torbeyns, A., Marcus, R.N., McQuade, R.D. and Carson, W.H. (2009) Aripiprazole monotherapy in the treatment of acute bipolar I mania: A randomized, double-blind, placebo- and lithium-controlled study. Journal of Affective Disorders, 112, 36-49. doi:10.4088/JCP.v66n1002

[5] Simon, J.S. and Nemeroff, C.B. (2005) Aripiprazole augmentation of antidepressants for the treatment of partially responding and nonresponding patients with major depressive disorder. The Journal of Clinical Psychiatry, 66, 1216-1220. doi:10.4088/JCP.v66n1002

[6] Yang, K.C., Su, T.P. and Chou, Y.H. (2008) Effectiveness of aripiprazole in treating obsessive compulsive symptoms. Progress in Neuropsychopharmacology \& Biological Psychiatry, 32, 585-586. doi:10.1016/j.pnpbp.2007.10.009

[7] Sarkar, R., Klein, J. and Kruger, S. (2008) Aripiprazole augmentation in treatment-refractory obssive-compulsive disorder. Psychopharmacology, 197, 687-688. 
doi:10.1007/s00213-008-1091-1

[8] DeLeon, A., Patel, N.C. and Crismon, M.L. (2004) Aripiprazole: A comprehensive review of its pharmacology, clinical efficacy, and tolerability. Clinical Therapeutics, 26, 649-666. doi:10.1016/S0149-2918(04)90066-5

[9] Abilfy/aripiprazole (2004) Bristol-Meyers Squibb Company/Otsuka American Pharmaceutical Inc., New York.

[10] Tsai, J.F. (2006) Aripiprazole-associated seizure. The Journal of Clinical Psychiatry, 67, 995-996. doi:10.4088/JCP.v67n0619b

[11] Malik, A.R. and Ravasia, S. (2005) Aripiprazole-induced seizure. Canadian Journal of Psychiatry, 50, 186.

[12] Stahl, M.S. (2008) Stahl's essential psychopharmacology: Neuroscientific basis and practical applications, 3rd edition, Cambridge University Press, Cambridge.

[13] Alldredge, B.K. (1999) Seizure risk associated with psychotropic drugs: Clinical and pharmacokinetic considerations. Neurology, 53, S68-75.

[14] Dailey, J.W., Yan, Q.S., Mishra, P.K., Burger, R.L. and Jobe, P.C. (1992) Effects of fluoxetine on convulsions and on brain serotonin as detected by microdialysis in genetically epilepsy-prone rats. The Journal of Pharmacology and Experimental Therapeutics, 260, 533-540.

[15] Stahl, M.M., Lindquist, M, Pettersson, M., Edwards, I.R., Sanderson, J.H., Taylor, N.F., Fletcher, A.P. and Schou, J.S. (1997) Withdrawal reactions with selective serotonin re-uptake inhibitors as reported to the WHO system. European Journal of Clinical Pharmacology, 53, 163-169. doi:10.1007/s002280050357

[16] Cassano, G.B., Fagiolini, A., Lattanzi, L., Monteleone, P., Niolu, C., Sacchetti, E., Siracusano, A. and Vita, A. (2007)
Aripiprazole in the treatment of schizophrenia: A consensus report produced by schizophrenia experts in Italy. Clinical Drug Investigation, 27, 1-13. doi:10.2165/00044011-200727010-00001

[17] Duggal, H.S. and Mendhekar, D.N. (2006) High-dose aripiprazole in treatment-resistant schizophrenia. The Journal of Clinical Psychiatry, 67, 674-675. doi:10.4088/JCP.v67n0420c

[18] Gupta, S., Chohan, M. and Madhusoodanan, S. (2004) Treatment of acute mania with aripiprazole in an older adult with noted improvement in coexisting parkinson's disease. Primary Care Companion to the Journal of Clinical Psychiatry, 6, 50-51. doi:10.1345/aph.1H240

[19] Chavez, B. and Poveda, R.A. (2006) Efficacy with high-dose aripiprazole after olanzapine-related metabolic disturbances. The Annals of Pharmacotherapy, 40, 22652268. doi:10.1345/aph.1H240

[20] Preskorn, S.H. (2003) Relating clinical trials to psychiatric practice: Part I: The case of a 13-year old on aripiprazole and fluoxetine. Journal of Psychiatric Practice, 9, 307-313. doi:10.1097/00131746-200307000-00006

[21] Woolley, J. and Smith, S. (2001) Lowered seizure threshold on olanzapine. The British Journal of Psychiatry, 178, 85-86. doi:10.1192/bjp.178.1.85-a

[22] Beasley, C.M.Jr., Tollefson, G., Tran, P., Satterlee, W., Sanger, T. and Hamilton S. (1996) Olanzapine versus placebo and haloperidol: Acute phase results of the North American double-blind olanzapine trial. Neuropsychopharmacology, 14, 111-123. doi:10.1016/0893-133X(95)00069-P 